\title{
Reposicionamiento de marca: el camino hacia la competitividad de las pequeñas y medianas empresas
}

\author{
Rebranding: the road to competitiveness of small and medium businesses
}

\section{Cristian Hernández-Gil ${ }^{1}$ Edward Fabián Figueroa-Ramírez ${ }^{2}$ Luis Eduardo Correa-Corrales ${ }^{3}$}

Recibido: abril 26 de 2018 Aceptado: junio 28 de 2018

\section{Resumen}

Con la apertura de nuevos mercados basados en el conocimiento, cobra importancia el desarrollo de marcas que vayan a tono con el estilo de vida de cada consumidor, un reto para las pymes que siempre han subestimado su capacidad de construir su propia identidad corporativa. El objetivo de este documento es analizar cómo a través del reposicionamiento de la marca, se puede lograr la competitividad de las empresas sin importar el tamaño del negocio, solamente considerando la indagación constante del mercado. Se desarrolló un estudio descriptivo donde se definieron tres categorías de análisis: reposicionamiento como estrategia, la construcción de la marca y la competitividad en las pymes. Estas categorías se identificaron en 54 documentos, correspondientes a artículos científicos, libros y proyectos de grado recuperados de bases de datos académicas. Se concluye que reposicionar una marca amerita el diseño e implementación de sistemas de planeación, comunicación y monitoreo, en donde todas las áreas de la organización participan, con miras a enfocar sus actividades económicas y/o sociales en función de uno o varios mercados.

Palabras clave: reposicionamiento, marca, competitividad, pymes, mercado.

\section{Abstract}

The opening of new markets based on knowledge takes importance in the development of brands that are related to the lifestyle of the consumer. It is a challenge for the Pymes that have always underestimated their capacity to construct their own corporate identity. The aim of this paper is to analyze how through the repositioning of the brand, the enterprise competitiveness can be achieved no matter the size of the business, considering the constant investigation of the market. A descriptive study was developed in which three categories of analysis were defined: repositioning as a strategy, the construction of the brand and the competitiveness of Pymes. These categories were identified in 54 documents that correspond to scientific articles, books and thesis from academic databases. It is concluded that repositioning a brand demands the design and implementation of planning, communication and monitoring systems, in which all the areas of the organization participate in order to focus their economic and social activities in function of one or more markets.

Keywords: repositioning, brand, competitiveness, Pymes, market.

1 Administrador de Empresas, Especialista en Mercadeo Gerencial, Estudiante de Maestría en Ciencias de la Educación, Universidad de la Amazonia, Florencia, Colombia. E-mail: cris.hernandez@udla.edu.co

2 Estudiante de Administración de Empresas, Universidad de la Amazonia, Florencia, Colombia. E-mail: edw.figueroa@udla.edu.co

3 Estudiante de Administración de Empresas, Universidad de la Amazonia, Florencia, Colombia. E-mail: lui.correa@udla.edu.co 


\section{Introducción}

La marca es el elemento central de cualquier estrategia empresarial. Por ende, en la actualidad el reto de cualquier organización es aprender a construirla basándose en el desarrollo de una identidad corporativa holística, que incluye desde el diseño y elaboración de uno o varios productos hasta la comunicación efectiva y permanente con el mercado que va a cubrir (Del Río-Cortina, Cardona-Arbeláez \& Guacarí-Villalba, 2017). El mercado cada vez conoce más rápido sobre tendencias, asume cambios sin avisar y se impone cuando se trata de exigir; esto lleva a exageraciones como encontrar en un solo producto, bien o servicio, todo lo que se requiere, como en el caso de los teléfonos inteligentes. De ahí que el único elemento funcional para que una marca sea efectiva, es la orientación hacia el mercado. Si no existe esta condición es posible que la única salida sea el fracaso y un declive rotundo (desde el punto de vista del ciclo de producto).

Las pymes no son ajenas a este escenario, pues su papel en el desarrollo de los países latinoamericanos o emergentes es contundente y significativo. En Colombia, el $98 \%$ del aparato empresarial lo conforman pequeñas y medianas empresas (Revista Dinero, 2016). Esta consolidación se ha dado gracias al fenómeno de la globalización y a la permanencia de la sociedad del conocimiento, lo que las ha llevado a ampliar sus fronteras o radios de acción y volverse tan populares o productivas como las grandes empresas, diseñando e implementando estrategias de internacionalización para poder crecer y sobrevivir, hasta ser consideradas como entes competitivos. En este sentido, la competitividad se convierte en un llamado para que las empresas más preparadas y ágiles, no solo limiten sus fines a vender, sino que además generen una rentabilidad necesaria para resistir (Alcaide, Bernués, Diaz-Aroca, Espinosa, Muñiz \& Smith, 2013). Esto implica aprender a posicionarse de forma eficiente en los nuevos mercados regionales, dirigiéndose hacia los segmentos más atractivos y rentables.
Sin embargo, antes de posicionar o reposicionar una marca, las empresas deben aprender a construirla. Es aquí donde se tiene el primer cuello de botella de toda organización, pues se subestiman al no sentirse capaces de desarrollarse estratégicamente, considerando que no tienen los recursos o la capacidad adecuada para enfrentar el mercado. En vista de lo anterior, el objetivo de este documento es analizar cómo a través del reposicionamiento de la marca, se puede lograr la competitividad de las empresas sin importar el tamaño del negocio, solamente considerando la indagación constante del mercado.

\section{Materiales y métodos}

Se realizó un estudio descriptivo con enfoque cualitativo, donde inicialmente se revisaron cien documentos relacionados con el reposicionamiento de la marca y su influencia en la competitividad. Los documentos consultados correspondieron a 60 artículos entre académicos y revistas especializadas, 25 proyectos de grado, y 15 libros, obtenidos de bases de datos académicas, como: Academia.edu, Google Académico, Dialnet, Redalyc, Scielo, Elibro, Virtualpro, y Ebrary.

El resultado de este proceso fue la construcción de una matriz constituida por la información más relevante y propia de cada documento, a saber: resumen, año de publicación, autores, citas, objetivos y principales resultados, entre otros. La revisión final se adelantó sobre 54 documentos, así: 32 artículos, 15 libros y 7 proyectos de grado, que fueron seleccionados por su relación con el reposicionamiento de la marca, en función de la operatividad de las pequeñas y medianas empresas, pymes.

Luego se adelantó un proceso de relectura y clasificación de cada insumo documental, estableciendo tres categorías de análisis, a saber: reposicionamiento como estrategia, la construcción de la marca y la competitividad en las pymes. La discusión se centró en un análisis que dio como resultado el establecimiento de una relación de interdependencia entre estas tres categorías. 


\section{Resultados y discusión}

\subsection{La competitividad en las pymes}

La competitividad ha estado asociada con los indicadores del desarrollo empresarial pero muchas veces no se entiende su concepto o su alcance, en especial cuando las organizaciones se enfrentan a la tarea de alcanzarla. El concepto de competitividad, aunque guarda relación con el sentido de la competencia, no solo se asume desde la rivalidad entre competidores, sino que también se enfoca en la capacidad de los entes económicos o sociales para sostenerse en un mercado; esto permite un crecimiento constante, adquiriendo ventajas competitivas (diferenciadoras), a partir de las comparativas (sin innovación).

Pero la realidad es otra para las pymes. Según Gálvez y García (2012) este tipo de empresas, cuya orientación es hacia las ventas, generalmente no desarrollan planes de mercadeo, y por ende no tienen en cuenta la innovación, ni siquiera al diseño del producto; además, no tienen una imagen de marca propia, ni mucho menos cuentan con programas de servicio al cliente. Es así como, Man, Lau y Chan (2002) señalan que las organizaciones con mayor experiencia gestionando mercados, usualmente son competitivas, pues reacciona mejor ante las exigencias del mercado utilizando elementos como la calidad, el precio y el diseño. Esto no quiere decir que una empresa con una nueva marca no logrará dicha condición, pues para ello se tiene la gestión del mercadeo, un factor de éxito rotundo que permite la generación de valor y la consecución de un porcentaje gradual de participación en el mercado.

Rodríguez y Sandoval (2017) explican que al estar las empresas inmersas en la globalización no solo basta con la comercialización de productos de alta calidad para lograr la competitividad; adicionalmente se requiere la capacidad de ser identificadas por la sociedad, a través de una marca desde la cual se generen vínculos de tipo emocional y simbólico con los consumidores. Esta relación es más relevante que la simple transacción de compra y venta de bienes y servicios. Igualmente, aseguran que la gestión de marcas permite una mayor confianza en los colaboradores, un reconocimiento público y un aumento de su reputación y prestigio ante los demás stakeholders (grupos de interés).

En este sentido, en términos empresariales, la competitividad está definida por: la productividad, la rentabilidad, la posición competitiva, la participación en el mercado interno y externo, las relaciones interempresariales, el sector y la infraestructura regional. La disminución de competitividad en una empresa se reflejaría en los "indicadores financieros como producto de la disminución del nivel de ventas y su consecuente pérdida de participación en el mercado..." (Vanegas, Botero \& Restrepo, 2014)

En este documento el término competitividad se retoma de Diez (2008), quien se basa en el planteamiento de su modelo de "diamante" integrado por cuatro factores: las condiciones de los factores de producción; las condiciones de la demanda; las industrias relacionadas y de apoyo; y las estrategias de la empresa, estructura y rivalidad. Para luego deducir, que la competitividad desde un enfoque micro es la capacidad de una empresa u organización económica para incursionar en el mercado y conservarse por largo tiempo.

En este punto se establece la relación entre la competitividad y el desarrollo de la marca. Hernández, Uribe y Barrera (2018), en su trabajo comprobaron la relación existente entre las variables del Mix de la mercadotecnia y la competitividad. Según los autores, en el contexto de su investigación (Guadalajara, México), las pymes tienen que asegurarse a la marca (definiendo claramente las actividades de la mezcla de mercadeo) para que sus clientes tengan lealtad y logren la competitividad.

Desde el mismo enfoque, para la Agencia Digital Creativa Drinlike (sf), las marcas asumen gran importancia en los mercados caracterizados por los altos índices de competitividad ya que esta es un 
elemento definitivo de diferenciación, posicionamiento y rentabilidad. Suele suceder, que la mayoría de las organizaciones de pequeña escala inician su negocio con una visión inmediatista, de corto plazo, descuidando, por ejemplo, el prota- gonismo y la construcción de la marca. Es por ello que se hace un llamado al desarrollo de la misma, para que se puedan alcanzar las condiciones de competitividad, como se ilustra en la figura 1.

\begin{tabular}{|c|c|c|c|c|}
\hline Notoriedad & Posicionamiento & Comunidad & Permanencia & Ahorro \\
\hline $\begin{array}{l}\text { - Cuanto } \\
\text { más se repite } \\
\text { la imagen de } \\
\text { marca a lo } \\
\text { largo del } \\
\text { tiempo, mayor } \\
\text { es la } \\
\text { notoriedad o } \\
\text { presencia de } \\
\text { la empresa. }\end{array}$ & $\begin{array}{l}\text { - Factores } \\
\text { como símbolos, } \\
\text { valores, cultura, } \\
\text { proyecto son } \\
\text { aquellos que la } \\
\text { imagen de } \\
\text { marca refuerza } \\
\text { y transmite con } \\
\text { el fin de hacerle } \\
\text { un hueco a la } \\
\text { empresa en la } \\
\text { historia. }\end{array}$ & $\begin{array}{l}\text { - Una } \\
\text { imagen fuerte } \\
\text { crea un } \\
\text { sentimiento } \\
\text { de comunidad } \\
\text { entre los } \\
\text { clientes al } \\
\text { verse } \\
\text { identificados } \\
\text { con ella. }\end{array}$ & $\begin{array}{l}\text { - Mantener } \\
\text { una constante } \\
\text { evolución de la } \\
\text { imagen de la } \\
\text { marca teniendo } \\
\text { en cuanto a los } \\
\text { cambios en el } \\
\text { mercado, } \\
\text { permite } \\
\text { asegurarla } \\
\text { continuidad de la } \\
\text { empresa a lo } \\
\text { largo del tiempo }\end{array}$ & $\begin{array}{l}\text { - Una } \\
\text { imagen bien } \\
\text { establecida desde } \\
\text { el principio puede } \\
\text { suponer un gran } \\
\text { ahorro en el } \\
\text { presupuesto de la } \\
\text { empresa. No es } \\
\text { necesario } \\
\text { reinventar } \\
\text { constantemente la } \\
\text { imagen gráfica, } \\
\text { toda imagen está } \\
\text { disponible por } \\
\text { completo en la } \\
\text { política de marca. }\end{array}$ \\
\hline
\end{tabular}

Figura 1. Condiciones para alcanzar la competitividad.

Son muchas las estrategias de marketing que toda empresa puede utilizar para aumentar su crecimiento y competitividad. Las pymes deberían enfocarse en su mercado meta y no solo en el cercano, sino también a través de los avances tecnológicos, lograr expandirse y cruzar las barreras internacionales logrando cumplir las metas de la organización. La mayoría de las pymes no creen indispensable un departamento de marketing, pensando que, por ser pequeña o mediana, no es necesario. Lo que no se toma en cuenta es el hecho de que es el departamento de marketing el que define el consumidor al cual la empresa se enfoca y es éste quien define el proceso por el cual el cliente se verá atraído. Es este departamento el que motivará la innovación dentro de la empresa, en cuanto a producto, precio, plaza y promoción (Hernández, López \& Mora, 2016).

Otros autores destacan la visión estratégica del mercadeo como elemento esencial para el logro de estándares competitivos, siempre y cuando vaya de la mano de una investigación incesante del mercado. En su conjunto, los factores cultura- 
les y subculturales son condicionantes externos que tienen gran influencia en el comportamiento de consumo que producen el logro de mejores niveles de competitividad (Ávila, Moreno \& Ocegue$d a, 2018)$. Para sobrevivir y prosperar en un mercado altamente competitivo, distinguiéndose de los competidores, es necesario incrementar la efectividad del marketing en las empresas mediante el neuromarketing, conociendo lo que el cliente en realidad desea y no lo que dice que quiere, contando con una información adecuada para lograr la correcta planeación y distribución del producto y/o servicio en el mercado meta (Brooksbank, 2002).

Romero y Rodríguez (2014) aseguran que otra manera de lograr indicadores favorables de competitividad es el compromiso firme con la participación en el sector exterior, ya que la actividad internacional de las PYME refuerza su crecimiento, impulsa su nivel de competitividad e innovación y favorece la sostenibilidad de la compañía a largo plazo.

En este punto, la relación entre la competitividad, el mercadeo y la construcción de la marca empieza a tener sentido; ahora nos enfocaremos en el contexto de las economías emergentes. Para Andrade (2015) en Ecuador y en muchas regiones de Latinoamérica, el ímpetu de ganar una mayor participación de mercado ha infundado en muchas empresas, el desarrollo de la marca como un activo de gran importancia para la organización, para así cumplir con la tarea de ser competitivos. Las pymes no han sido apoyadas de manera adecuada en América Latina, debido a las políticas públicas deficientes, y a la personalidad empresarial de sus propietarios. Aunque el apoyo por parte del sector público mejoraría la competitividad de las pymes y sus posibilidades de éxito en el mercado nacional y global, esto no debería ser camisa de fuerza para lograrla (Olmedo-Carranza, 2011).
En ocasiones se observa como las empresas de pequeña escala, culpan al gobierno por no apoyarlas económicamente, es decir a través de subsidios o de créditos condonables. Enríquez, Adame y Heredia, (2017), citando a Bonales, explican que se debe retomar el concepto de competitividad sistémica, que se caracteriza por reconocer que un desarrollo industrial exitoso no se logra meramente a través de una función de producción en el nivel micro, o de condiciones macroeconómicas estables, sino también por la existencia de medidas específicas del gobierno y de organizaciones privadas de desarrollo orientadas a fortalecer la competitividad de las empresas. Es decir, lograr la competitividad es un trabajo sinérgico en donde se presumen la participación no egoísta de todos los actores económicos.

Sin embargo, Martínez y Javier (2017), revelan que en Colombia existen diversas debilidades de carácter estructural que limitan la competitividad de estas organizaciones, reduciendo su capacidad de supervivencia y crecimiento, lo cual lleva a muchas de ellas al declive. Estos autores establecen la necesidad de realizar estudios transversales para analizar el comportamiento de las pymes, frente a las políticas que se desarrollen como consecuencia de la implementación de los acuerdos de paz, así como la reacción de la economía nacional ante esta situación. Adicionalmente, la posibilidad de fortalecer las políticas de competitividad e innovación se convierten en un factor de diferenciación que puede favorecer el crecimiento de las organizaciones, concluyen los autores.

En este sentido, la triada: competitividad, marketing y construcción de marca deben ir de la mano de un elemento transversal, asociado con la información y los datos. Según Soto (2006), la gestión del conocimiento comprende diversas actividades, ver figura 2. Si una organización emprende un viaje hacia la identificación y desarrollo de algunos de esos elementos, asume coherentemente la gestión del conocimiento para la efectividad de su competitividad. 


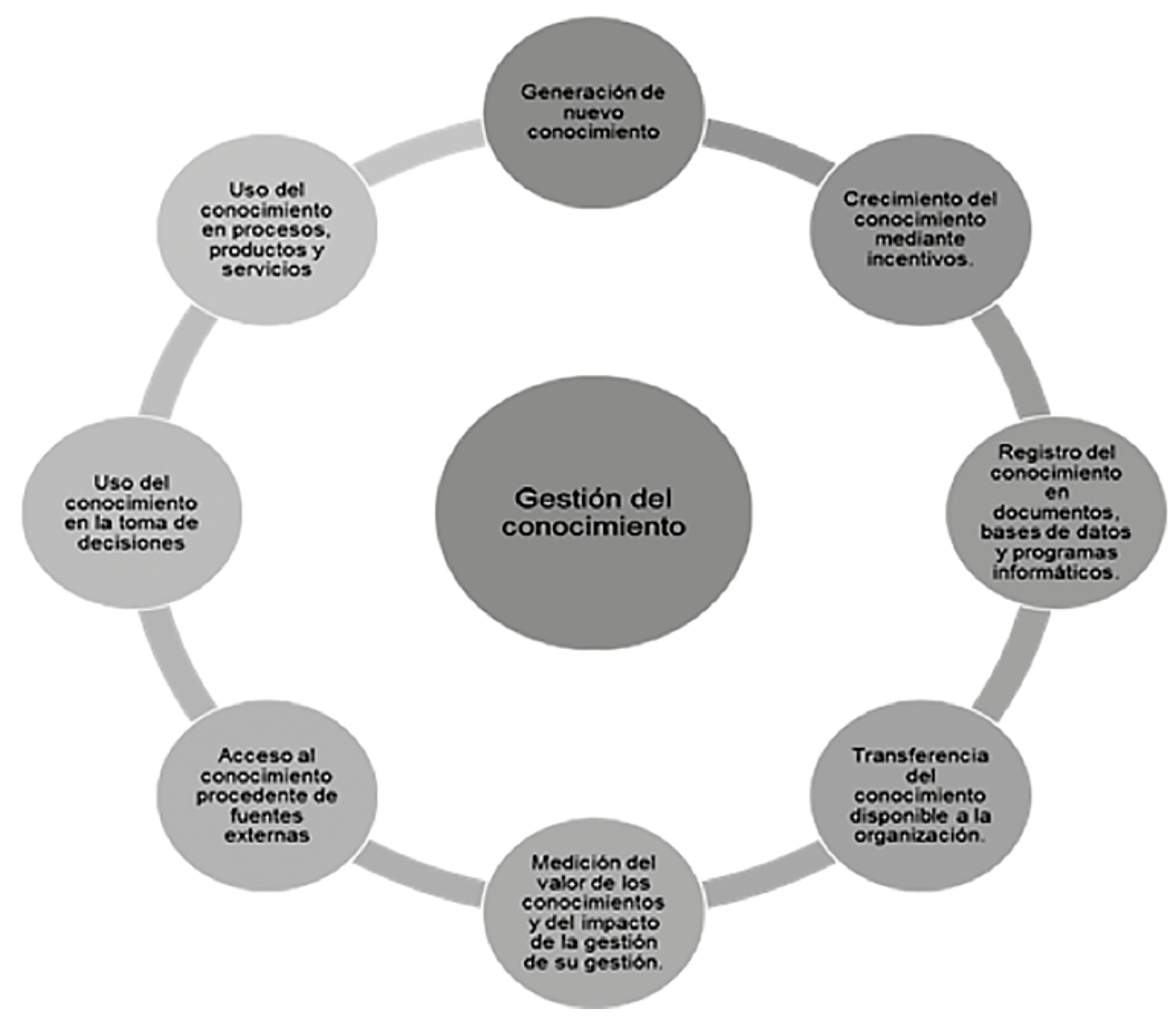

Figura 2. Actividades de la Gestión del Conocimiento.

\subsection{La construcción de la marca}

Antes de construir una marca, es necesario definir qué es y cómo esta les da valor a los clientes y permite la consecución de los objetivos dentro de una organización. El término no es del todo nuevo. Cruz, Chávez y Pérez (2017) reconocen que hasta principios de los noventa se involucraron conceptos como reconocimiento de marca, conocimiento de marca y asociaciones de marca, debido a que los directivos de mercadotecnia en esa época poseían una mentalidad técnica (una orientación exclusiva hacia las ventas o la producción).

Según Puma, Quimi, y Zea (2017), para crear una marca se debe tener clara la diferencia entre un nombre comercial y una marca. Un nombre propio no es una marca, un nombre es útil para individualizar el producto. El nombre de marca es la prime- ra oportunidad que tiene un producto para decir algo de sí mismo al mercado objetivo. Así mismo, las funciones de conocimiento de marca se resumen en hacerla accesible (conoce de su existencia) para el consumidor, proporcionar reconocimiento, recuerdo, familiaridad (aceptabilidad y preferencia) e intención de compra para hacer frente a la competencia, ocupando una posición superior en la mente del consumidor (top of mind) mediante una marca notoria (Gonzáles \& Rodríguez, 2012).

Es así como se empieza a hablar de gestión de marcas o branding. Este consiste en transmitir a productos y servicios, el poder de una marca, esencialmente mediante la creación de factores que los distingan de otros productos y servicios. "Los especialistas en marketing deben mostrar a los consumidores "quién" es el producto (dándole un nombre y empleando otros elementos de marca 
para ayudarles a identificarlo), así como qué hace y por qué deberían adquirirlo". (Kotler \& Keller, 2015, p. 321). Las organizaciones que consiguen tener marcas de éxito, es porque no las consideran recursos tácticos sino activos estratégicos de primer orden. Una marca bien gestionada es una fuente potencial de ingresos. En la medida que la marca genera "deseabilidad" se puede decir que genera mayor demanda y por ende más precio (Alcaide et al., 2013).

En este sentido, se ha demostrado que las marcas podían ser caracterizadas por descriptores de personalidad, tales como: joven, colorido y suave. Estas características permiten a los consumidores expresarse a sí mismos o sus ideales. Este simbolismo de la personalidad de marca es de gran importancia, ya que "las personas son seres sociales que se construyen en la sociedad a través de significados y las marcas ayudan muchas veces a construir el ser social que las personas quieren ser" (Goñi, 2013). Es por eso que, Fromm y Garton (2013) afirman que el Santo Grial, el gran desafío o secreto, para muchos marketeros (encargado del área de mercadeo), hoy en día, es crear una marca que no pierde vigencia, "que le pueda hablar a todos los consumidores, sin importar la generación y el momento de sus vidas."

Lo anterior significa que construir una marca implica conocer adecuadamente al consumidor. Por ejemplo, Fernandini (2017), considera que los MiIlennials son un segmento difícil de capturar cuando una marca no se acerca hacia ellos con temas de interés. El autor reconoce que a ellos no se les puede abordar hablándoles de lo que a la empresa como marca le interesa, sino debe hablárseles de lo que a ellos les importa, sus tendencias, para luego introducir la marca y el producto dentro de él, dándole siempre la posibilidad de sentir que la marca se mueve con ellos y los escucha; pero no basta solo con escucharlos, sino también responderles y hacerlos sentir importantes. Igualmente, Gutiérrez (2015), explica que uno de los factores más importantes a tener en cuenta en la gestión de una marca es la percepción del consumidor, ya que, a partir de las ideas que este se forma de la marca, se desprenden comportamientos y afinidades que influyen en la relación que puede llegar a tener y transmitir a su entorno y a su decisión de compra.

Es aquí donde cobra importancia la estrategia. Andrade (2015) explica que una empresa de cualquier tamaño que no trabaja bajo una línea de administración estratégica, tiene casi garantizado su fracaso. Todas las pymes deben tener orientación al mercado (OM) porque de aquí parte la implementación del concepto de Marketing y sus correspondientes estrategias a favor de las empresas de nueva creación. Se debe considerar el desarrollo estratégico si se requiere construir una marca.

"La construcción de una marca es el resultado de la gestión general y la capacidad de la estrategia para ajustarse a las condiciones de un entorno cambiante, en función de las necesidades del consumidor. La innovación y la gestión de la experiencia de consumo son determinantes". (Revista Dinero, 2012).

Hablar de marca implica ser estrategas como organización y ser gestionadores de conocimiento, definiendo las características e ideales de los usuarios del mercado. Barón, Villalba, y Toro (2017) recalcan la percepción de la innovación para garantizar, por un lado, aumento en las ventas, y por otro, la optimización de la relación con el cliente. Este comportamiento refleja una tendencia usual en el sector del microempresariado, donde son las ventas el objeto principal donde se dirigen todas las estrategias, incluso las de innovación.

Klyver (2016), considera tres tipos de objetivos distintos para las pymes. Por un lado, están los objetivos de comunicación, como por ejemplo lograr engagement con el target objetivo; por otro, los de branding, como lograr reconocimiento de marca; y para finalizar, los de comercialización, como el clásico objetivo de impulsar la venta de un producto o servicio determinado. Así, es indispensable insistir en que las relaciones públicas son el 
pilar actual en el cual las empresas, de cualquier tamaño, fundamentan la actividad de sus estrategias de comunicación para generar el posicionamiento de una marca (Fernández, 2006).

Adicionalmente, Pérez (2012) presume un nuevo aspecto dentro de la gestión de marcas y es el uso de redes sociales, en las pymes, las cuales permiten medir el impacto comunicacional que estas empresas pueden lograr en sus mercados locales. Se analiza el nivel de comprensión de la imagen mediante la relación real de atributos generales y específicos de la empresa por usuarios de las principales redes sociales. De esta forma se logrará medir indicadores de lealtad de la marca, la cual está en "el corazón del valor de todas las marcas, porque una vez obtenida, la lealtad es persistente" (Lara, Saltos, Mayorga, Carvajal \& Moreno, 2017).

Otro aspecto relacionado con la marca es la percepción de las características de los productos desde la óptica del consumidor. Kapferer (1992), define la calidad percibida basada en un nivel de confianza y constituye un componente esencial de valor de marca, convirtiéndose en uno de los factores más importantes y determinantes en la toma de decisión final al adquirir o no un producto.

Gálvez, Stalin, Bermeo y Guerrero (2015), concluyen que el proceso de creación de una marca es un proceso extenuante que debe empezar con la correcta definición del proyecto, organización, producto o servicio que se va a trabajar. Esto implica dos momentos: En primer lugar, se debe establecer el público al cual se dirige; es necesario diferenciar cada público de interés para establecer estrategias y acciones de comunicación acordes para cada uno de ellos. En segundo lugar, se deben tener en cuenta los códigos o sistemas de comunicación; así se lograría identificar si es pertinente transferir el mensaje al receptor por medio auditivo, visual, gustativo, olfativo o por el tacto.

Paris (2014) asume que para interpretar un significado en la mente del consumidor se debe partir del significado base en la misma mente del consu- midor o del código cultural en la mente del mercado. Y esto solo se logra identificando los elementos funcionales del bien o servicio que lleva la marca. Desde el punto de vista funcional, un producto tiene múltiples componentes que desempeñan roles diferentes para la satisfacción de las necesidades de los usuarios. En este sentido, existen al menos cuatro niveles funcionales de un producto, a saber: básico, mejorado, aumentado y emocional.

Lo anterior lleva a considerar que el proceso de la gestión de marcas tiene también el objetivo de transmitir la cultura y filosofía de la empresa. Los estudios usualmente se encaminan a impregnar la marca desde una total categorización de la compañía, pero no se ocupan de las herencias ancestrales o las competencias innatas de la zona para generación de ventaja competitiva (Cruz, Saltos, \& Garces, 2017). El trabajo dentro de la organización, para construir una marca, debe ser holístico y sinérgico, donde todos los colaboradores de la organización participen. Rocha, y Alberto (2015), explican que para iniciar el liderazgo de marca se necesita que la empresa sea reconocida por la vinculación de sus cualidades o características específicas de gestión. La marca de liderazgo se construye fuera de la empresa, supliendo las necesidades de los clientes, y también de manera interna, con las expectativas de los inversores y la motivación de su cliente interno.

\subsection{Reposicionamiento como estrategia}

Antes de hablar de reposicionamiento debemos entender en qué momento una marca ha sido posicionada, para partir de la necesidad de que la misma logre nuevas formas de ser visualizada por el consumidor, reposicionándose. Para ello es importante reconocer que el mundo y el mercado se han transformado. "Los consumidores han cambiado sus hábitos en los últimos años volviéndose más y más volubles, impredecibles y cada vez más inmunes a las herramientas de comunicación de marketing tradicional" (Wohfeil \& Whelan, 2005). 
En este caso se consideran las definiciones de Trout y Ries (2000). El posicionamiento se refiere a "Lo primero que viene a la mente cuando se trata de resolver un problema de cómo lograr ser escuchado en una sociedad sobre comunicada". El posicionamiento no es lo que se realiza con un producto, sino lo que se construye en la mente de las personas; es decir, se posiciona el producto en la mente de las personas.

Posicionar una marca implica, primero que todo, el desarrollo de experiencias inmersas en la mente del consumidor. Brakus, Schmitt y Zhang (2008) hablan de dichas experiencias y las consideran siempre como algo subjetivo, ya que en la mayor parte se desarrollan en la mente y son el resultado no sólo de uno, sino de múltiples puntos de contacto entre la marca y su audiencia. Así mismo, Soto-del Blanco (2015) define que antes de diseñar y hacer vivir la experiencia, cada empresa debe observar dónde se encuentra la marca dentro de la mente del consumidor y buscar qué es lo que se está haciendo bien y qué mal. Si está desarrollando aspectos negativos o diferentes a los que la compañía desea trasmitir, es el momento de reposicionar.

Por otra parte, Stefany y Borghini (2015) asumen el reposicionamiento como el cambio de la imagen corporativa, definición de una planeación estratégica de la marca, diseño de la cartera de negocios, estrategia para establecer relaciones con los clientes y la transmisión de los valores por los canales de distribución. Esto quiere decir que el éxito en los negocios no pasa por ganar la batalla por la preferencia de marca sino la guerra por la relevancia de marca a través de una oferta innovadora que logre una diferenciación sostenible (Aaker, \& Álvarez-del Blanco, 2015).

Rivas (2017), argumenta que: la pérdida de participación de mercado y la disminución de ventas son indicios de la pérdida de la credibilidad hacia una marca por parte de los consumidores, proponiendo generar una nueva posición mental como la mejor vía de solución. El reposicionamiento invo- lucra mejora en algunos aspectos; por ejemplo, en el caso del yogurt, los valores que sobresalen son el gusto, la cantidad de azúcar, grasa, o disminución de determinados ingredientes, esto cuando se trata de productos; mientras que, en servicio, por ser intangible, involucra calidez, amabilidad, respeto, agilidad, entre otros aspectos que son calificados por los consumidores al momento de la compra (Vinces \& Rivera, 2017).

De acuerdo con Ruíz y Grande (2016) “La clave para conseguir el reposicionamiento es el cambio en la variable comunicación. Por consiguiente, el cambio de imagen comienza con una redefinición del target". Esto conlleva a considerar que el reposicionamiento requiere de estudios que permitan que los productos o servicios regresen a ocupar un lugar en la mente del consumidor, agregando valores y atributos que antes no tenían y que es necesario resaltarlos para que los consumidores acepten y consuman (Santos, 2016), o por otro lado a definir una nueva estrategia de segmentación, en donde el foco será un nuevo nicho de mercado.

"Es importante que la marca realice una correcta segmentación del mercado, adaptando su estrategia y centrándose en un segmento de consumidores con un conocimiento del sector algo más alto que la media y con una clara predilección, por ejemplo, por el lujo. Esto permitirá desarrollar un mix de marketing adaptado a las características y deseos de los consumidores" (Lloret, 2017, p. 31).

Andrade, Mendoza y Pulido (2017), introducen un nuevo concepto: el rebranding. Aseguran que este se genera debido a dos causas: i) la propia adaptación de la marca a la evolución de los gustos de los consumidores, las tendencias seguidas por la competencia o las exigencias de los marcos regulatorios; ii) relacionada al reposicionamiento, a la necesidad de cambiar una opinión sobre la marca o empresa que se ha desviado de los objetivos planteados, por ejemplo, tras una crisis de reputación. Adicionalmente, Ramaswamy (2008), explica que el reposicionamiento también va de la mano 
con los procesos cocreativos, siendo un elemento importante para generar un diálogo constructivo; es decir, una interacción a través del compromiso y la reciprocidad entre la marca (empresa), y el consumidor (mercado).

Lo anterior se ilustra en la marca Tuenti, que nació en 2006 como una red social, pero cuatro años más tarde dejó de lado ese negocio para convertirse en un operador móvil virtual. En ese momento comenzó un proceso de reposicionamiento de percepciones para conseguir que el público identificara a Tuenti como un operador móvil y no como una red social. Analizando el posicionamiento comunicativo de Tuenti se ha podido confirmar que la compañía no empleó los mismos atributos y características para definirse a lo largo del tiempo de manera consistente y reposicionarse en el mercado (Muñoz, Herrero, \& Guardia, 2017). Esto permite establecer que una estrategia de posicionamiento comunicativo debe estar basada en los principios de objetividad y orientación al mercado, para obtener a lo largo del tiempo una posición en la mente del cliente prospecto y situar al producto o marca como opción única y diferenciada respecto a la competencia (Porter, 2012).

En últimas, se debe tener cuidado con el seguimiento a los indicadores de posicionamiento de una marca, pues aunque sean excelentes, la marca puede encontrarse en una situación no deseada. Por ejemplo, en el 2008 se descubrió que una de las marcas más icónicas del mundo, de lencería para damas, fue considerada demasiado sexy (connotación sexual), y había alcanzado una sensualidad equivocada, no deseada; esta situación llevó a la marca a tener un mal desempeño en las ventas 2007" (Merryck, 2008, citado por Hoyos, 2016).

\subsection{Discusión}

Lo descrito en los apartados anteriores permite establecer que, antes de reposicionar una marca, es necesario haberla posicionado, e inclusive, siguiendo la lógica, haberla construido. Es por este enfoque sistemático que las empresas, especialmente las pymes, deben desarrollarse para lograr la competitividad.

La competitividad no debe ser vista, solamente, como el liderazgo en el mercado, sino además, como la gestión efectiva del conocimiento empresarial y de mercado, que hace que la orientación hacia el consumidor logre sus frutos (ventaja competitiva). La marca construida debería ser vista como un ejemplo a seguir, con la cual la gente se identifica, se relaciona, con la que vibra, se apasiona y se fideliza en el largo plazo.

En ocasiones, este proceso puede tornarse complejo si no se actúa de manera estratégica y creativa. Es aquí donde la organización desde su principio holístico debe apreciar a su cliente interno y trabajar de la mano con él para que, con la innovación, la marca de los productos o de la empresa se esté renovando de acuerdo a las necesidades de quienes la compran y la consumen.

La cuestión de posicionar o reposicionar una marca no necesariamente requiere de altos niveles de consumo de recursos. Hoy por hoy, el know how de una organización, es decir la capacidad intelectual y creativa de sus colaboradores es prácticamente suficiente para convertir productos en grandes marcas, y que estas logren adquirir la condición humana necesaria para que el consumidor, cliente, influenciador o usuario logre conectarse y la adhiera a su estilo de vida. Además, el uso de redes sociales, y herramientas tecnológicas de prueba son tan útiles para conectar al consumidor con el mensaje creativo, el cual es el reto que todo ente tiene y que solo puede ser producto de la invención del trabajo en equipo.

\section{Conclusiones}

Existen muchos caminos para lograr la competitividad de las pymes, si de reposicionar marca se trata. Entre los tres más importantes se tienen: la cocreación, la aplicación de los niveles funcionales del producto y la inclusión de la responsabili- 
dad social dentro de la estrategia empresarial. Si se trata de cocrear, la pyme debe lograr un acercamiento eficaz y suficiente con el consumidor y crear una red de información integral, en la cual se comunique con el mercado y escuche desde su perspectiva cómo visualizan el futuro de la marca. Esto generalmente se realiza a través de laboratorios de innovación empresarial o ciudadana utilizando la metodología del design thinking.

Por otro lado, si se utiliza la aplicación de los niveles funcionales del producto, la gerencia debe enfocar sus esfuerzos en una planeación estratégica eficiente que promueva, con la participación de los colaboradores, espacios y momentos de innovación en miras a fortalecer los niveles más complejos (el ampliado y el emocional), los cuales salen en gran medida del radio de acción del objeto social del ente. Y si lo que se busca es usar una connotación más amigable con el entorno, se puede reposicionar la marca desde el uso estratégico de la responsabilidad social, elemento importante hoy en día y más cuando se trata de reducir impactos ambientales, generando conciencia para establecer y vivir en un mundo mejor.

En consecuencia, desde la academia, es necesario continuar con el desarrollo de líneas de investigación en mercadeo y comportamiento del consumidor. La investigación permitirá proporcionar elementos que, a través de lo indagado, brinden herramientas para que las empresas incorporen nuevos conocimientos que mejoren el cumplimiento de su función económica y social.

En síntesis, reposicionar es una actividad permanente que apoya a las acciones misionales de una organización. Estamos pasando por épocas donde reina la incertidumbre, en especial con el acceso a una verdadera información del mercado, el cual cada día es más cambiante, complejo y de opiniones divergentes y sentimientos de "bipolaridad". Aunque a pesar de todo, si se hace bien la tarea y se llega a una gran aproximación, los resultados frente al ser competitivos serán óptimos: aumento en ventas, rentabilidad maximizada y perdurabilidad del valor en el mercado.

\section{Referencias}

Aaker, D., \& Álvarez-del Blanco, R. (2015). Las marcas según Aaker. Empresa Activa.

Agencia Digital Creativa Drinlike (sf). ¿Por qué las empresas necesitan una imagen de marca fuerte? Recuperado de: http://www.drimlike.com/es/ blog/por-que-las-empresas-necesitanuna.html

Alcaide, J. C., Bernués, S., Diaz-Aroca, E., Espinosa, R., Muñiz, R., \& Smith, C. (2013). Marketing y pymes: Las principales claves de marketing en la pequeña y mediana empresa. Marketing y pymes Ebook. Recuperado de: https://goo.gl/wVmkJQ

Andrade-Perdomo, J. G., Mendoza-Peralta, J. A., \& Pulido-Medina, C. A. (2017). Diagnóstico y rebranding de la marca Edime (Proyecto de grado). Universidad de Bogotá Jorge Tadeo Lozano. Recuperado de: https://goo.gl/kNrK4c

Andrade, J. P. (2015). El aporte del marketing en la creación del capital de marca en las pymes de Manabí. ECA Sinergia, 6 (1), 77-91. Recuperado de: https://revistas.utm.edu.ec/index.php/ECASinergia/ article/view/244/203

Ávila, G. V., Moreno, T. E. N., \& Ocegueda, J. L. F. (2018). Efectos del neuromarketing en las ventas directas en las pymes joyeras de Jalisco. Una perspectiva no paramétrica. Red Internacional de Investigadores en Competitividad, 10 (1). Recuperado de: https://riico.net/index.php/riico/article/ view/1301/971

Barón-Pulido, M., Villalba, J., \& Toro, M. (2017). ¿Qué transmite su marca? Estrategias para innovar desde la comunicación. Recuperado: http://190.131.241.186/handle/10823/806

Brakus, J.J., Schmitt, B.H. \& Zhang, S. (2008). Experiential Attributes and Consumer Judgments, in 
Schmitt, B.H. y Rogers, D.L. Handbook on Brand and Experience Management, 174 - 187. Masachuset, USA: Edwar Elgar Publishing.

Brooksbank, R., \& Taylor, D. (2002). The adoption of strategic marketing and its contribution to the competitive success of New Zealand companies. Marketing Intelligence \& Planning

Cruz, J. G. S., Chávez, N. I. S., \& Pérez, L. F. L. (2017). La notoriedad de marca y el posicionamiento en pymes de Tungurahua: un estudio de construcción categorial teórica. Revista Publicando, 4 (11 (2)), 668-681. Recuperado de: https://www.rmlconsultores.com/revista/index.php/crv/article/ view/618/pdf_428

Cruz, J. G. S., Saltos, A. C. L., \& Garces, L. E. G. (2017). La identidad de marca desde el criterio del consumidor ecuatoriano. Revista Publicando, 4 (11 (2)), 463-479. Recuperado de: https://www.rmlconsultores.com/revista/index.php/crv/article/ view/585/pdf_406

Del Río-Cortina, J., Cardona -Arbeláez, D., \& Guacarí-Villalba, A. (2017). Responsabilidad social empresarial y construcción de la marca: una nueva mirada a las estrategias de gestión. Revista de Investigación, Desarrollo e Innovación, 8 (1), 49-60. doi: https://doi.org/10.19053/20278306. v8.n1.2017.7370

Diez, J. (2008). Organizaciones, redes, innovación y competitividad territorial: el caso Bahía Blanca. REDES-Revista Hispana para el Análisis de Redes Sociales, 14 (3). Recuperado de: http://www.raco.cat/ index.php/Redes/article/viewFile/115236/144110

Enríquez, L. A., Adame, M. G., \& Heredia, J. L. H. (2010). Gestión del conocimiento a través de los componentes del capital intelectual y su incidencia en la competitividad de las pymes en Aguascalientes. Red Internacional de Investigadores en Competitividad, 4 (1). Recuperado de: https://www. riico.net/index.php/riico/article/view/808/735
Fernández, S. A. (2006). Las relaciones públicas como elemento tangencial entre la publicidad, propaganda, información y otras áreas de la comunicación. Revista mexicana de ciencias políticas y sociales XL-VII (196): 159-170. Recuperado de: http://www.redalyc.org/html/421/42119610/

Fernandini-Figari, M. (2017). Estrategia de reposicionamiento para lograr el crecimiento de una marca de bebidas de chocolate. Recuperado de: http:// repositorioacademico.upc.edu.pe/upc/handle/10757/622820

Fromm, J., \& Garton, C. (2013). Marketing to Millenials. Reach the largest and most Influential Generation of Consumers Ever. 1st Edition. AMACOM.

Gálvez, A., Stalin, W., Bermeo-Pacheco, J. A., \& Guerrero-Jirón, J. R. (2015). Conceptos introductorios sobre Branding. Recuperado de: http://repositorio. utmachala.edu.ec/handle/48000/6708

Gálvez, E. J., \& García, D. (2012). Impacto de la innovación sobre el rendimiento de la mipyme: Un estudio empírico en Colombia. Estudios Gerenciales, 28 (122), 11-27. doi: https://doi.org/10.1016/ S0123-5923(12)70191-2

Gonzáles, M., \& Rodríguez, T. (2012). Imagen de marca y product placement. Madrid, España: ESIC Editorial.

Goñi-Avila, N., Torres-Moraga, E., \& Aguilera-Valenzuela, S. (2013). Dimensiones de la personalidad de la marca en México. Revista de Ciencias Sociales (Ve), XIX (2), 213-225. Recuperado de: http://www. redalyc.org/html/280/28026992002/

Gutiérrez, L. M. R. (2015). Estudio de cambio en la percepción del consumidor de marcas top of mind en el mercado colombiano. Poliantea, 10 (18), 113-142. doi: http://dx.doi.org/10.15765/plnt. v10i18.535

Hernández, A. D., López, J. D. J. U., \& Mora, J. A. F. (2016). El impacto que tiene el marketing sobre las 
pymes manufactureras de la industria metalmecánica para mejorar su competitividad. Red Internacional de Investigadores en Competitividad, 9 (1), 149-169. Recuperado de: https://www.riico.net/ index.php/riico/article/view/10/10

Hernández, A. D., Uribe, E. G. G., \& Barrera, M. A. O. (2016). Estrategias de Marketing y ventajas competitivas en las pymes mueblera. Red Internacional de Investigadores en Competitividad, 10 (1), 238253. Recuperado de: https://riico.net/index.php/ riico/article/view/1315/984

Hoyos, R. B. (2016). Branding el arte de marcar corazones. Bogotá, Colombia: Ecoe Ediciones. Recuperado de: https://goo.gl/ZngeWG

Kapferer, J. (1992). La marca, capital de la empresa. Principios y control de su gestión. Bilbao: Deusto.

Klyver, C. J. (2015). Las Redes Sociales y las pymes. Una relación productiva. Cuadernos del Centro de Estudios de Diseño y Comunicación, 16 (57), 169180. Recuperado de: http://fido.palermo.edu/servicios_dyc/publicacionesdc/archivos/555_libro. pdf\#page $=169$

Kotler., \& Keller. (2015). Dirección de Markeitng. México: Pearson Educación

Lara-Flores, E. A., Saltos-Cruz, J. G., Mayorga-Díaz, M. P., Carvajal-Larenas, R. P., \& Moreno-Gavilánez, K. A. (2017). La gestión de marca, un factor estratégico de competitividad en pymes. Revista CienciAmérica, 6 (1), 64-84. Recuperado de: http://cienciamerica.us/openjournal/index.php/uti/article/ view/74/60

Lloret, A. D. (2017). Análisis de posicionamiento de marca en el sector automovilístico (Tesis doctoral). Recuperado de: https://riunet.upv.es/handle/10251/89482

López-Rodríguez, C., \& Perea-Sandoval, J. (2017). El gerente de marca como protagonista de las orga- nizaciones en Colombia. Equidad Y Desarrollo, (28), 259-278. doi: https://doi.org/10.19052/ed.4179

Man, T. W. Y., Lau, T., \& Chan, K. F. (2002). "The competitiveness of small and medium enterprises: $A$ conceptualization with focus on entrepreneurial competencies". Journal of Business Venturing, 17 (2), 123-142. doi: https://doi.org/10.1016/S08839026(00)00058-6

Martínez, A., \& Javier, O. (2017). Futuro de las pymes colombianas en el postconflicto (Proyecto de grado). Universidad Militar Nueva Granada.

Muñoz, C. F., Herrero, E. M., \& Guardia, M. L. G. (2017). Principios básicos de posicionamiento comunicativo. Análisis del caso de Tuenti (20122015). Revista ICONO14, 15 (2), 49-69. doi: https:// doi.org/10.7195/ri14.v15i2.1000

Olmedo-Carranza, B. (2011). Políticas de apoyo a las PYMES en América Latina: Entre avances innovadores y desafíos institucionales. Problemas del desarrollo, 42 (167), 193-195. Recuperado de: http://www.scielo.org.mx/scielo.php?script=sci_arttext\&pid=S0301-70362011000400011\&ln$\mathrm{g}=\mathrm{es} \& \mathrm{t} \operatorname{lng}=\mathrm{es}$

Paris, J. A. (2014). ¿Cuántos significados tiene un producto?. Revista Caderno Profissional de Marketing-UNIMEP, 2 (1), 1-14. Recuperado de: https:// www.cadernomarketingunimep.com.br/ojs/index.php/cadprofmkt/article/view/16/22

Pérez-Amezcua, M. (2012). Redes sociales, mecanismos generadores de reputación organizacional para las PyMEs. Universidad \& Empresa, 14 (22), 131 150. Recuperado de: https://revistas.urosario.edu. co/index.php/empresa/article/view/2004/2050

Porter, M. E. (2012). Estrategia competitiva: Técnicas para el análisis de los sectores industriales y de la competencia. Madrid, España: Pirámide.

Ramaswamy, V. (2008) "Co-creating value through customers' experiences: the Nike case". Strat- 
egy \& Leadership, 36 (5), 9-14, doi: https://doi. org/10.1108/10878570810902068

Revista Dinero (2012). Top of mind. Recuperado de: http://www.dinero.com/edicion-impresa/caratula/ articulo/top-of-mind/148440

Revista Dinero (2016). Mipymes generan alrededor del $67 \%$ del empleo en Colombia. Recuperado de: https://www.dinero.com/edicion-impresa/pymes/ articulo/evolucion-y-situacion-actual-de-las-mipymes-en-colombia/222395

Rivas Pincay, D. A. (2017). Plan de marketing para reposicionar la marca besitos fiesta, ciudad de Guayaquil, año 2017 (Proyecto de grado). Guayaquil, Ecuador.

Rocha, M., \& Alberto, J. (2015). Estrategias sólidas para estructurar pymes y organizaciones nacionales desde la alta gerencia (Proyecto de grado). Universidad Militar Nueva Granada.

Rolando-Arellano, C. (2010). Marketing: Enfoque América Latina El marketing científico aplicado a Latinoamérica. Primera edición. Editorial Pearson - Prentice Hall.

Romero, I., \& Rodríguez-Gutiérrez, M. (2014). Sobre la internacionalización de la PYME y su contribución a la imagen exterior de España. Comillas Journal of International Relations, 0 (1), 91-104. doi: https://doi.org/10.14422/cir.i01.y2014.007

Ruíz, S., \& Grande, I. (2016). Comportamientos de compra en el consumidor. Madrid, España: Bussines \& Maketing ESIC.

Santos, D. d. (2016). Tácticas aplicadas del marketing. Madrid, España: Mapcal.

Soto, M., \& Barrios, N. (2006). Gestión del conocimiento: Parte I. Revisión crítica del estado del arte. ACIMED, 14 (2). Recuperado de: http:// scielo.sld.cu/scielo.php?script=sci_arttext\&pi$\mathrm{d}=$ S1024-94352006000200004\&Ing=es\&tlng=es.

Soto-del Blanco, A. (2015). Estrategias de reposicionamiento de marca y su capacidad para incidir en las percepciones de los consumidores: análisis de Mercedes Benz. Recuperado de: http://uvadoc.uva. es/handle/10324/14160

Stefany, H. G., \& Borghini, N. (2015). Recorte y delimitación del tema del PG. Recuperado de: http:// fido.palermo.edu/servicios_dyc/blog/alumnos/ trabajos/946_731.pdf

Trout, J., \& Ries, A. (2000). Posicionamiento. Madrid, España: McGraw Hill.

Vanegas, G., Botero, C., \& Restrepo, A. (2014). Una aproximación mediante lógica difusa al análisis de la competitividad empresarial. Administration $Y$ Organizations, 17 (33), 14. Recuperado de: https:// goo.gl/V4LhPa

Villarroel-Puma, M., Carranza-Quimi, W., \& Cárdenas-Zea, M. (2017). La creación de la marca y su incidencia en el posicionamiento de un producto. Revista Publicando, 4 (12 (2)), 684-697. Recuperado de: https://www.rmlconsultores.com/revista/index.php/crv/article/view/734

Vinces-Alvarado, A. Y., \& Rivera-Baldeón, M. M. (2017). Reposicionamiento de yogurt kiosko para aumentar la participación de mercado en las cadenas de autoservicios (Proyecto de grado). Guayaquil, Ecuador.

Wohfeil, M., \& Whealn, S. (2005). Consumer motivations to participate in marketing - events: the role of predispositional involvement. European Advances in Consumer Research, 7, 125 - 130. 